\title{
Effect of Somatic Experiencing Resiliency-Based Trauma Treatment Training on Quality of Life and Psychological Health as Potential Markers of Resilience in Treating Professionals
}

\section{OPEN ACCESS}

Edited by:

Wolf E. Mehling,

University of California, San Francisco,

United States

Reviewed by:

Herbert Jelinek,

Charles Sturt University, Australia

Marlysa Sullivan,

Maryland University of Integrative Health, United States

*Correspondence:

Neal E. Winblad nwinblad@nwinblad.com

Specialty section:

This article was submitted to Autonomic Neuroscience,

a section of the journal

Frontiers in Neuroscience

Received: 15 October 2017

Accepted: 29 January 2018

Published: 16 February 2018

Citation:

Winblad NE, Changaris M and Stein PK (2018) Effect of Somatic

Experiencing Resiliency-Based

Trauma Treatment Training on Quality of Life and Psychological Health as

Potential Markers of Resilience in

Treating Professionals.

Front. Neurosci. 12:70.

doi: 10.3389/fnins.2018.00070

\begin{abstract}
Neal E. Winblad ${ }^{1 *}$, Michael Changaris ${ }^{2}$ and Phyllis K. Stein ${ }^{3}$
${ }^{1}$ Private Pracitce Truama and Developmental Trauma Specialist, Pleasanton, CA, United States, ${ }^{2}$ Health Psychology Department, Integrated Health Psychology Training Program, The Wright Institute, Berkeley, CA, United States, ${ }^{3}$ Heart Rate Variability Laboratory, Cardiovascular Division John T. Milliken Department of Medicine, School of Medicine, Washington University in St. Louis, St. Louis, MO, United States
\end{abstract}

Background: Individuals who treat trauma are at significant risk of vicarious traumatization and burnout. Somatic Experiencing ${ }^{\circledR}\left(\mathrm{SE}^{\circledR}\right)$ is a resiliency-focused trauma treatment modality designed to address autonomic nervous system (ANS) dysregulation and its impacted physical health and mental health symptoms e.g., anxiety, depression, post-traumatic stress disorder, migraines, fibromyalgia, and chronic fatigue, etc. The $\mathrm{SE}^{\circledR}$ training supports the development of clinical skills to reduce physical health/mental health symptoms as well as increase clinician resilience. Individuals who display resilience often have increased experiences of well-being (quality of life) and decreased levels of self-reported psychological symptoms. Greater resilience could mitigate the risks to providers and the clients they treat.

Materials and Methods: This within-groups, longitudinal study assessed students $(N=18)$ over the course of a 3 -year $S E^{\circledR}$ practitioner training. This training focuses on increased ANS, physical, and emotional regulation skills. The convenience of a web-based survey allowed for: measures of a general quality of life (WHOQOL-BREF), psychological symptoms, somatic, anxiety, and depressive symptoms (PHQ-SADS), as well as a measure of early life exposure to adversity (CDC/Kaiser Permanente ACE Score Calculator Questionnaire). The clinician survey was conducted yearly for 3 years. Future studies would do well to also include laboratory-based objective measures of ANS functioning.

Results: ANOVA with repeated measures showed that there were significant reductions in anxiety symptoms (GAD7, $p<0.001$ ) and somatization symptoms (PHQ15, $p<0.001$ ). Health-related quality of life (a measure of physical well-being) and social quality of life (a measure of interpersonal well-being) both increased significantly (Health QoL $p=0.028$; Social QoL $p=0.046$ ).

Conclusions: Results suggest that professionals attending the 3-year $\mathrm{SE}^{\circledR}$ training course experience a significant improvement in self-reported measures associated with 
resiliency including: quality of life (well-being) and psychological symptoms (anxiety and somatization). Our results support the importance of future research in a larger sample and support the exploration, cross-sectionally and prospectively, of the relationship of clinician resiliency and changes in clinician resiliency with $S E^{\circledR}$ training and clinical outcomes. These data have implications for other professions at risk of exposure to vicarious trauma (VT) including nurses, medical providers, and paramedics.

Keywords: Somatic Experiencing ${ }^{\circledR}$, resiliency, vicarious traumatization, compassion fatigue, burnout, quality of life, ANS dysregulation, traumatic stress

\section{INTRODUCTION}

Individuals who treat traumatized and other highly dysregulated clients are at higher risk of developing symptoms of burnout, compassion fatigue and vicarious traumatization/secondary trauma (Sprang et al., 2007; Craig and Sprang, 2010). Professionals who develop these symptoms are less effective in their work, need to seek their own treatment, and can experience long-term negative health outcomes (Pearlman and Saakvitne, 1995; Baird and Jenkins, 2003; Trippany et al., 2004; Craig and Sprang, 2010). Professionals who treat PTSD may also be required to work in areas and situations that could increase their own chances of exposure to traumatic events, e.g., war zones or areas with high community violence (Johnson et al., 2014).

Richardson and Waite (2002) define resiliency as "a selfrighting force within everyone that drives him/her to pursue selfactualization, altruism, wisdom, and harmony with a spiritual source of strength." Others define resilience as the ability to adapt to adversity (Horn et al., 2016). Li et al. (2016) demonstrated a correlation between social support, hope, resilience, and quality of life in bladder cancer patients, with resilience, hope and social support accounting for $30 \%$ of the variance in quality of life. Resilience and hardiness has been found to mitigate the effects of trauma exposure, improve neuroendocrine functioning and improve coping (Tugade and Fredrickson, 2004; Ménard et al., 2016).

Clinicians who develop the capacity to manage, reduce and mitigate impacts of stress-based injuries may be more effective at treating PTSD and other symptoms of intense dysregulation in both their offices and in the field (Horn et al., 2016; Pereira et al., 2016). Somatic Experiencing ${ }^{\circledR}$ (SE) is a trauma treatment modality that works with trauma through supporting innate resilience and reducing stress, as well as stress-based injuries. A growing body of research has demonstrated improvements in multiple symptom domains as a result of $\mathrm{SE}^{\circledR}$ treatment (Leitch, 2007; Whitehouse and Heller, 2008; Andersen et al., 2017; Brom et al., 2017).

\section{Trauma, Burnout, and Vicarious Trauma}

Rates of burnout, secondary traumatic stress (STS) and vicarious trauma (VT) are high among treating professionals. Increased resilience, social support and coping strategies can mitigate both risk and impact of exposure (Bell et al., 2003). Secondary traumatic stress (STS) is a traumatic reaction that can be catalyzed from a single exposure to another individual's experience of a traumatic event, whereas VT is a result of cumulative exposure to traumatic experiences over time. Thus, among therapists, VT is triggered by a cumulative exposure to traumatic experiences of patients/clients and refers to harmful changes in how a professional views their work, themselves, their world and other professionals (Pearlman and Saakvitne, 1995; Baird and Jenkins, 2003). Both STS and VT can impact clinicians who work with individuals exposed to trauma and can affect their functioning and lead to an array of stress reactions.

Estimates of rates of STS among psychotherapist working with trauma in military settings are as high as $19.2 \%$ (Cieslak et al., 2013). Research into VT among nursing and medical providers has identified a prevalence of between 26 and $40 \%$ of individuals experiencing symptoms due to their work experiences. Child protective services workers, for example, were found to have a $35 \%$ rate of VT in one study (Sabin-Farrell and Turpin, 2003).

Understanding the basic principles of risk and resilience in treating professionals and developing self-regulation/emotion regulation capacity could improve functioning and reduce risk of VT (Pearlman and Saakvitne, 1995; Baird and Jenkins, 2003; Pereira et al., 2016). Risk factors for both STS and VT include a history of previous traumatic events. The temporal exposure to client traumatic experiences, e.g., caseload, hours with clients, percent of trauma clients in caseload, and amount of exposure over time has also been related to risk for both VT and STS in some studies (Pearlman and Saakvitne, 1995). Perception of coping has been found to mitigate symptoms of both VT and STS (Pearlman and Saakvitne, 1995).

While many programs aimed at increasing resilience focus on cognitive coping, a recent study found some significant limitations to top-down cognitive coping strategies. In this recent study, in the general population, cognitive regulation (using cognitive coping) alone was found to have a limited impact on cortisol, a stress hormone dysregulated in PTSD (Pereira et al., 2016). These data point to the need for the inclusion of additional non-cognitive coping capacities for effective clinician resilience training.

The costs of VT and STS are significant for the individual, the people they treat and the families of the trauma treating professional (Baird and Jenkins, 2003; Cocker and Joss, 2016). Developing strategies for improved resilience among treatment providers could help mitigate these costs. 


\section{Clinician Resilience and Clinical Outcomes}

Many factors have been shown to be associated with clinician resilience. Some of these are: cognitive coping strategies, mindfulness, ability to access social support, and effectiveness of self-care. Mindfulness has been associated with reduced symptoms of PTSD and increased resilience in multiple populations. Fisher and Ogden (2009) developed the term "somatic mindfulness" to describe the ability to increase interoceptive awareness of bodily states and self-regulatory capacity in her work on Sensorimotor Psychotherapy ${ }^{\mathrm{TM}}$. In other research, clinician mindfulness was found to be related to better clinical outcomes. A 2016 study of mindfulness and clinician resilience found that to the degree that an individual practiced mindful self-awareness, such as meditation, they had improved clinical outcomes (Pereira et al., 2016). The Pereira study reviewed 37 therapists' caseloads for a total of 4,980 cases. A significant proportion of the clinician/therapist factor in effective clinicians was due to mindfulness and resilience. These data indicated that for less severe cases (mild to moderate depression) mindfulness alone improved clinical outcomes, but in the more severe cases (severe depression) the combination of mindfulness and resilience affected outcomes. In somatic therapies, such as Somatic Experiencing ${ }^{\circledR}$ and Sensorimotor Psychotherapy ${ }^{\mathrm{TM}}$, both increased clinician resilience and somatic mindfulness are core parts of training and interventions.

\section{Somatic Experiencing ${ }^{\circledR}$ and Resilience}

Somatic Experiencing ${ }^{\circledR}\left(\mathrm{SE}^{\circledR}\right)$ is a resiliency-based approach to trauma treatment that, rather than focusing on pathology, focuses on working with innate resilience and increasing capacity (Payne et al., 2015). SE ${ }^{\circledR}$ uses a bottom up approach that works with interoceptive awareness, affective states, and limbic activation. $S E^{\circledR}$ differs from many cognitive therapies which work to change cognitions in order to change affective states. $\mathrm{SE}^{\circledR}$ interventions are designed to help guide the client to increased contact with their bodily sensations (interoceptive cues and kinesthetic/muscular awareness), instead of focusing on cognitions. This bottom up approach is based on the fact that core aspects of trauma are housed in systems which emerge from brain structures deep below the cortex. As van der Kolk (1984) showed, in his initial studies of traumatized patients, central language centers of the brain (i.e., Brocca's area) are often shut down during reactions to trauma triggers. As a result, there is some significant aspect of the traumatic experience that is housed in what van der Kolk describes as "wordless terror" (van der Kolk, 1984).

Somatic Experiencing ${ }^{\circledR}$ works with what Payne et al. (2015) paper call the Core Response Network (CRN). The CRN includes subcortical, limbic system, motoric pathways, interoceptive cues, and basic arousal systems [i.e., the autonomic nervous system (ANS), the hypothalamic-pituitary-adrenal axis and the reticular activating system]. Dysregulation in these protective systems leads to the development of symptoms of trauma and other negative health outcomes. The $\mathrm{SE}^{\circledR}$ modality works by supporting the re-establishment of the innate regulatory capacity of the CRN via interoception (a core component of mindfulness), self-protection, emotion regulation, and self-awareness (Payne et al., 2015). To support the goal of re-establishing innate regulatory capacity (e.g., resilience), interventions are conducted in a client-centered, titrated manner.

While increased awareness and tolerance for affective states is a core outcome of $\mathrm{SE}^{\circledR}$ therapy, it is not primarily an exposure therapy. Rather than evoking intense traumatic memories directly, $S E^{\circledR}$ works indirectly and gradually with these memories, identifying resources and corrective shifts in states that lead to new interoceptive experiences that in turn lead to changes in the felt experience of safety, power, and competence. While $S E^{\circledR}$ shares many components with traditions such as meditation, Qigong, and yoga, it also has a specific set of tools that help clinicians and clients address the fundamental dysregulation of the CRN that underlies trauma (Payne et al., 2015). Parker et al. (2008) showed that tsunami victims in southern India showed $90 \%$ improvement in symptoms even at eight month follow up to short 75 minute treatment sessions using $\mathrm{SE}_{\mathrm{R}}$ skills.

\section{Somatic Experiencing ${ }^{\circledR}$ Training and Resilience}

The Somatic Experiencing ${ }^{\circledR}$ Practitioner training course is open to all practitioners who come into contact with clients struggling with the symptoms of trauma. It has three beginning modules (4 days each), three intermediate modules (4 days each), and two advanced modules ( 6 days each). In the beginning year students develop, through practice, their own resilience and are encouraged to practice self-regulation throughout the training. Students also learn the basic biology of trauma and resilience, core clinical skills for guiding and improving interoceptive awareness and integration of protective strategies mobilized to protect the body during a traumatic event. In the intermediate year students learn to address specific types of trauma, e.g., falls, motor vehicle accidents, natural disasters, violence, etc. In the advanced year, students learn skills for working with the biological syndromes that are often comorbid with trauma such as fibromyalgia, migraines, and IBS. Throughout the training and between training modules, participants are encouraged to practice self-regulation skills, work in consultation groups, and have regular sessions that support their own development of selfregulation capacity and increase their skill as trauma treating therapists. They are also encouraged to read and explore the literature of trauma, trauma healing, and self-regulation. It is expected that in addition to becoming skillful as therapists in treating trauma, these practices will lead to the increased ability to self-monitor, regulate affect, cope with stress, stay in mindful awareness, and improved resilience, although ours is the first study to explicitly test this hypothesis.

\section{Aim}

While the most traditional definition of resilience is the ability to withstand and rebound from adversity, in a study involving human subjects it would be unethical to create severe adversity in subjects lives in order to study their immunity to adversity. We therefore looked at secondary indicators of resilience in this study, which can be measured on self-report psychological measurement instruments. 
The purpose of this study was to test the effect of Somatic Experiencing ${ }^{\circledR}$ Training on measures of resiliency by assessing changes in clinician resilience longitudinally over the 3 year $S E^{\circledR}$ training course. Clinician quality of life, psychological symptoms and degree of early life trauma were measured. We used the World Health Organization Quality of Life-Brief (WHOQOL-BREF) to assess changes in well-being in four domains: psychological quality of life (mental wellness), health related quality of life (overall physical well-being), social quality of life (quality of social relationships), and environmental quality of life (measure of wellness on community and access to housing etc.,). Changes in psychological symptoms was measured using the Patient Health Questionnaire (PHQ-SADS), which includes three subscales for anxiety (GAD-7), somatic symptoms (PHQ-15), and depression (PHQ-9). In order to assess the early life adversity on changes in resilience, early life adversity was measured using the $\mathrm{CDC} /$ Kaiser Permanente $\mathrm{ACE}^{1}$ Score Calculator Questionnaire.

\section{METHODS}

\section{Recruitment}

Due to multiple challenges with sampling and randomization, a convenience sample of volunteer participants in the $\mathrm{SE}^{\circledR}$ 3year training course was used. Subjects were recruited from the cohort of students participating in the Somatic Experiencing ${ }^{\circledR}$ training course at five different sites: Berkeley, CA, Columbus, $\mathrm{OH}$, Chapel Hill, NC, NYC, NY, and Austin, TX. Individuals who attend the Somatic Experiencing ${ }^{\circledR}$ training course are an interdisciplinary group of health care providers including: psychologists, social workers, medical doctors, psychiatrists, physical therapists, and other body oriented therapists. During the Beginning I module of the $S E^{\circledR}$ training course, the lead teaching assistant read a recruitment script for the experimental group. Potential subjects volunteered by signing up and the lists of volunteers were sent to the principal investigator.

\section{Experimental Design}

This study was a within-groups pre-to post-test design. The within-groups methodology and multiple points of measurement reduce some of the variance found in between-groups designs. Study volunteers were assessed on changes over the course of 3 years on four variables: quality of life, physical symptoms, psychological symptoms, and at the initial measurement the effect of adverse childhood experiences. Beginning and intermediate training modules are 4 days ( $24 \mathrm{~h}$ in length), the advanced training year modules are 6 days $(36 \mathrm{~h}$ of continuing education). As shown in Figure 1, there were four points of measurement: at the start of $\mathrm{SE}^{\circledR}$ training course (beginning I), after the beginning year (end of beginning III, after the intermediate year (end of intermediate III), and after

${ }^{1}$ Centers for Disease Control and Prevention: Adverse Childhood Experiences (ACE) Study. Available online at: http://www.cdc.gov/ace/index.htm completion of the training course (after year three advanced level II).

\section{Outcome Measures WHOQOL-BREF}

The WHOQOL-BREF has four quality of life domains: psychological quality of life (PSYCH-QOL or mental wellness), health related quality of life (HEALTH-QOL or overall physical well-being), social quality of life (SOCIAL-QOL or quality of social relationships), and environmental quality of life (ENVIRONMENTALQOL or measure of wellness on community and access to housing etc.) (The WHOQOL Group, 1995; Power, 1998).

\section{PHQ-SADS}

The PHQ-SADS is a validated measure of stress, anxiety, somatic symptoms, and depression. (Han et al., 2009; Kroenke et al., 2010a,b; Kocalevent et al., 2013). The PHQ-SADS includes a question about distress and three subscales measuring anxiety (GAD-7), somatic symptoms (PHQ-15), and depression (PHQ-9).

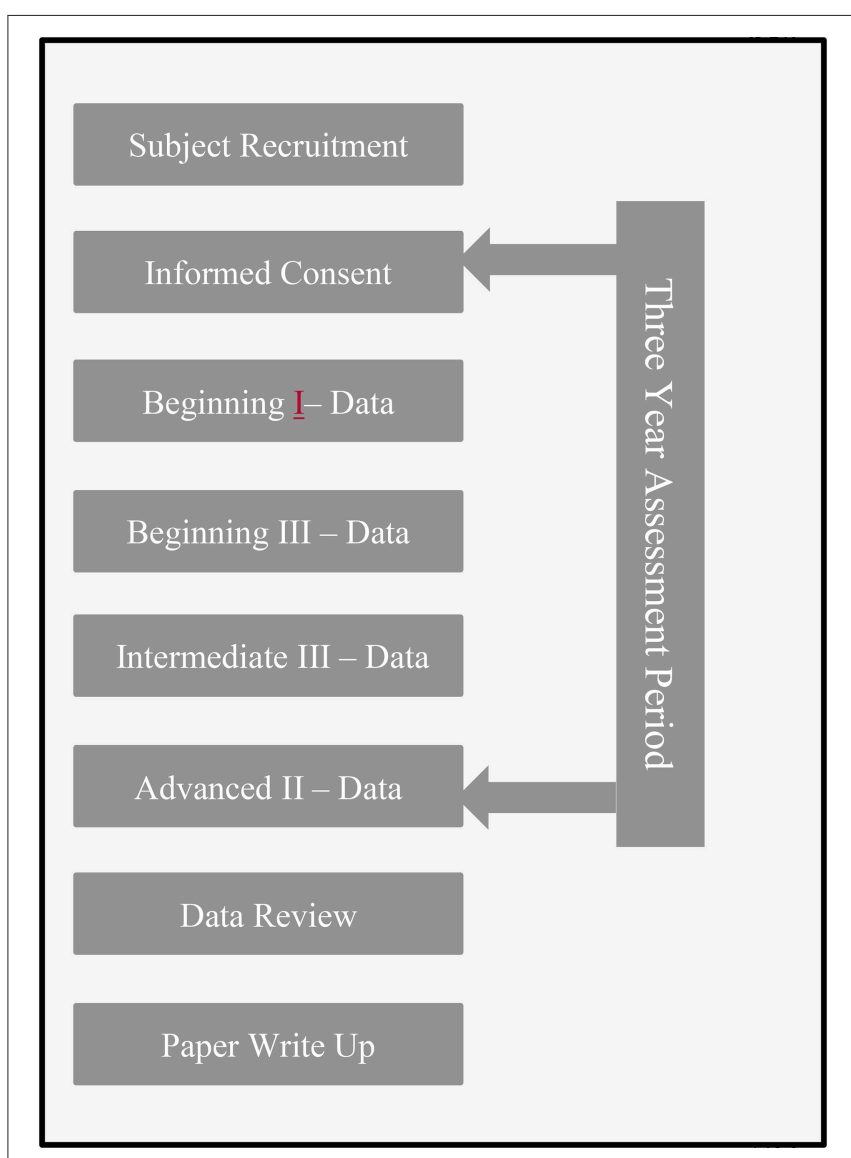

FIGURE 1 | Study timeline. This figure displays the study timeline including informed consent and data collection points. Data was collected over the course of the 3-year training period. 


\section{Data Collection}

Participants were contacted by email and given the URL for the web-based survey built around Limesurvey open source software (Engard, 2009). The survey included a short algorithm for calculating a subject identification number, such that the online data were de-identified before data entry and results were completely de-identified.

The web survey site asked participants to agree to the terms and conditions after reading the recruitment statement. It then collected some generic demographic information, followed by the survey questions. Participants were asked to send an email to the principal investigator acknowledging that they had completed the survey and informing him which cohort they were a part of. The PI tracked the training module schedule for each participant, and 2 weeks after the relevant modules, the PI sent emails to each study participant informing them that it was time to retake the survey. The participants would then take the survey and send an email to the PI announcing that they had completed this round of the survey. If no response was received a second email notice was sent.

\section{Statistical Methods}

ANOVA with repeated measures was used to test the hypotheses that students who completed the 3 years $\mathrm{SE}^{\circledR}$ training would show an increase in each of the four domain measures of quality of life measured using the WHOQOL BREF during the training and would also have decreases in symptoms of anxiety and somatic symptoms compared to baseline (PHQ-SADS) during the course of the training. Post-hoc analysis used Fischer's least significant difference (LSD) to assess differences between periods of measurement (i.e., years of the study) and changes in specific variables. Software was SPSS 23.0. Statistical significance was set at $p=0.05$.

\section{RESULTS}

\section{Participants}

$N=16$ participants self-identified as female and $N=2$ as male. $N=12$ participants self-identified as middle class and $N=5$ as upper class. None reported being lower SES.

\section{Participant Flow}

Initially $N=45$ participants signed up. After completion of the training, $N=18$ remained in the study. Per participant report, dropout was largely due to attrition in the training for financial reasons, needing to postpone finishing the training, loss of interest in $\mathrm{SE}^{\circledR}$, and no longer wishing to participate in the survey.

\section{Adverse Events}

To date there have been multiple studies exploring SE's impact on PTSD and other health/mental health challenges published in peer reviewed journals (Heller and Heller, 2004; Leitch, 2007; Leitch et al., 2009; Changaris, 2010). No significant adverse events were reported as a result of conducting these studies in any of these and none occurred in the current study.

\section{Detailed Results WHOQOL-BREF}

$N=15$ participants had complete data for the WHOQoL-BREF. As shown in Table $\mathbf{1}$ and Figures 2, 3, significant improvements in quality of life measures were observed in two of the four domains of the WHOQoL-BREF, Health-QoL ( $p=0.028)$, and Social-QoL $(p=0.046)$. Health improved significantly between the initial measurement and each of three subsequent measurements. Social-QOL increased significantly at the fourth measurement compared to each of the prior measurements. There were changes in Psychological and Environmental QoL but analysis showed them not to be significant.

\section{PHQ-SADS}

$N=18$ participants had complete data for the PHQ-SADS. As shown in Table 2 and Figures 4, 5, significant reduction in Anxiety (GAD-7) and Somatic (PHQ-15) symptoms was observed ( $p \leq 0.001$ for each). In general, scores improved between each training year. In post-hoc analysis, all periods of measurement were significantly different between first and follow up measures. The only non-significant changes were between the third (intermediate III) and fourth (advanced II) measurements. The PHQ-SADS also includes a single question that measures overall distress from the anxiety, somatic, and depression symptoms reported. Scores on the overall symptom distress question were significantly reduced between initial measurement (post beginning I) and final measure (post advanced II) training years $(p<0.001)$. Depression, as measured by PHQ9, could not be assessed due to an error in the question design in the survey platform leading to insufficient data.

TABLE 1 | Changes in Quality of Life (QOL) as Measured by the WHOQOL-BREF ( $N=15)$.

\begin{tabular}{|c|c|c|c|c|c|}
\hline & Beginning I & Beginning III & Intermediate III & Advanced II & $p$ for model \\
\hline HEALTH-QOL & $15.4 \pm 2.4$ & $16.2 \pm 0.1^{*}$ & $16.5 \pm 2.1^{*}$ & $16.3 \pm 2.4^{\star}$ & 0.028 \\
\hline PSYCH.-QOL & $14.4 \pm 2.0$ & $14.5 \pm 2.5$ & $15.3 \pm 2.2$ & $15.6 \pm 2.0$ & NS \\
\hline ENVIRONMENTAL-QOL & $15.5 \pm 2.5$ & $15.7 \pm 2.7$ & $16.2 \pm 2.1$ & $16.4 \pm 1.9$ & NS \\
\hline
\end{tabular}

*Significantly increased compared to Beginning $I, p \leq 0.033$

${ }^{\star}$ Significantly lower compared to Advanced II, $p \leq 0.025$. 


\section{PHQ-SADS Graphic Display of Data ACE Scores}

ACE scores were available at each time point for 15 participants. Self-reported ACE scores were not significantly different over the four assessments. No relationship was found between any of the measurements at Beginning I and ACE scores, due likely to the small sample size and the small number of subjects with high ACE scores.

\section{DISCUSSION}

Rates of community exposure to trauma are extremely high, in some cases $>60 \%$ of the population (Felitti et al., 1998; Anda et al., 2009). This has caused leaders in the field to call trauma the "hidden epidemic of our era." The ability of health practitioners to address symptoms of trauma in their health and mental health care systems could have a profound impact on multiple

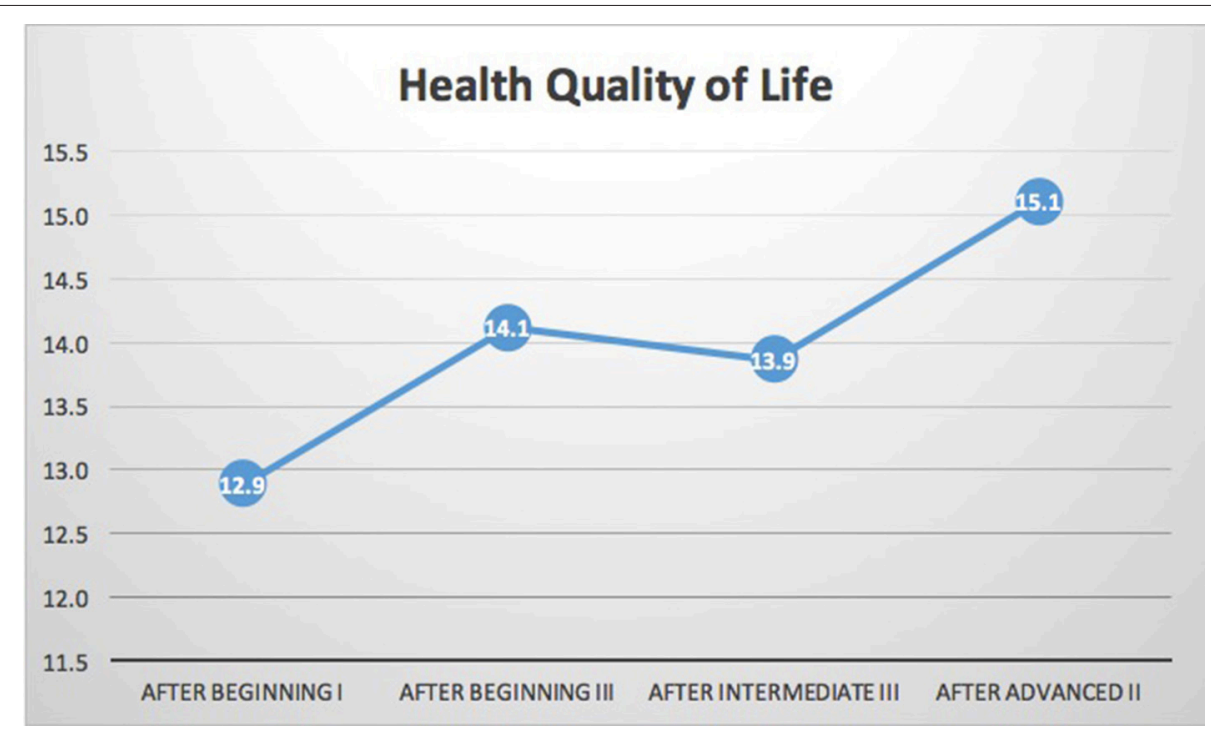

FIGURE 2 | WHOQOL-BREF health related quality of life. Results indicated significant improvement in health related quality of life $(p=0.028)$ on the WHOQOL-BREF. While the sample size is too small to clearly identify trends it is notable that there was a flattening of the trend line during the intermediate training year i.e., between the final beginning class (Beginning III) and final intermediate class (Intermediate III). Intermediate year training focuses on addressing specific types of traumas and focuses less on training of emotional regulation skills. It is possible that the exposure to learning about specific traumatic events may lead to a short-term level of distress that is reduced in the final two advanced year trainings.

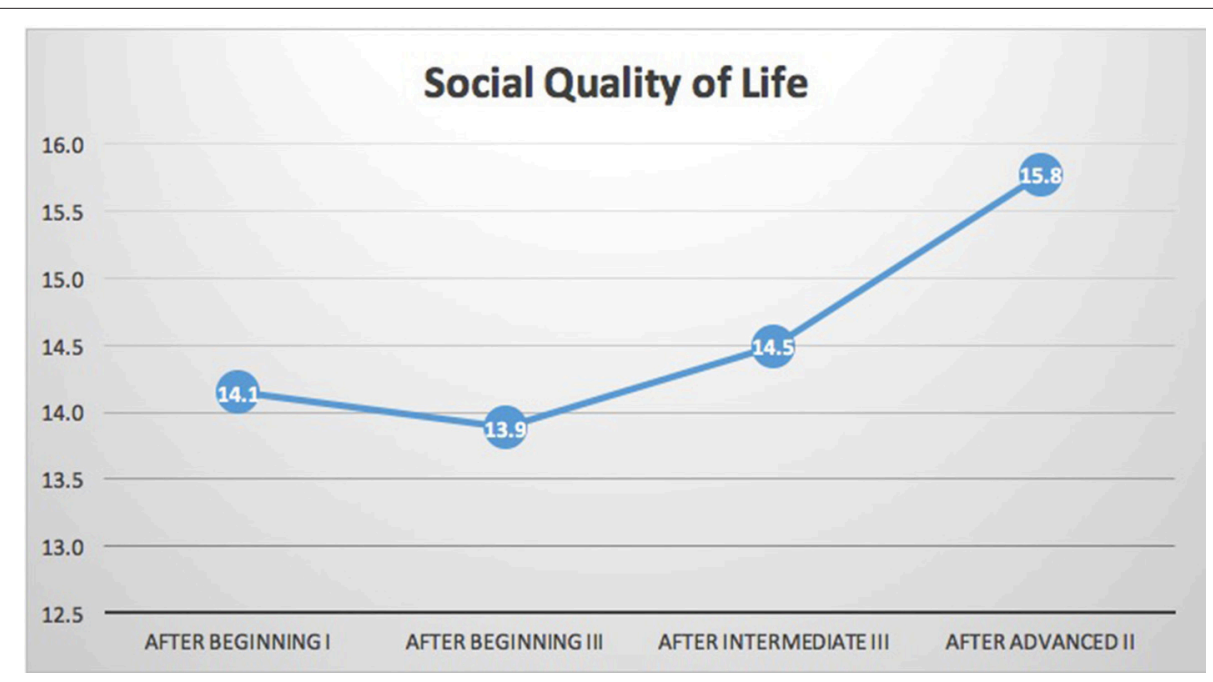

FIGURE 3 | WHOQOL-BREF social quality of life. Results indicated significant improvement in social quality of life $(p=0.046)$ on the WHOQOL-BREF. The trend in social quality of life was flat over the course of the three beginning modules. As above the sample size is too small to clearly identify trends. The beginning training year has several aspects that would address social quality of life. One of the core modules that could impact social quality of life is somatic boundaries and trauma. This module focuses on interpersonal safety and setting limits to support self-regulation. It could be that these skills require more significant practice before they can impact one's social connections or social quality of life. Alternatively, social quality of life may have a lag time from skill increase to change in behavior due to relationships being built over time. 
TABLE 2 | Changes in PHQ-15 and GAD-7 as Measured by the PHQ-SADS $(N=18)$.

\begin{tabular}{|c|c|c|c|c|c|}
\hline & Beginning I & Beginning III & Intermediate III & Advanced II & $p$ for model \\
\hline PHQ-15 (Som.) & $7.8 \pm 3.1$ & $6.1 \pm 3.1^{\star}$ & $4.9 \pm 3.5^{\star}$ & $3.8 \pm 2.4^{\star}$ & $<0.001$ \\
\hline GAD-7 (Anx.) & $5.5 \pm 4.6$ & $3 \pm 3.2^{\star}$ & $2 \pm 2.3^{\star}$ & $1.5 \pm 2.4^{\star}$ & $<0.001$ \\
\hline
\end{tabular}

*Significantly decreased compared to Beginning I, $p \leq 0.013$. NS for Intermediate III vs. Advanced II.

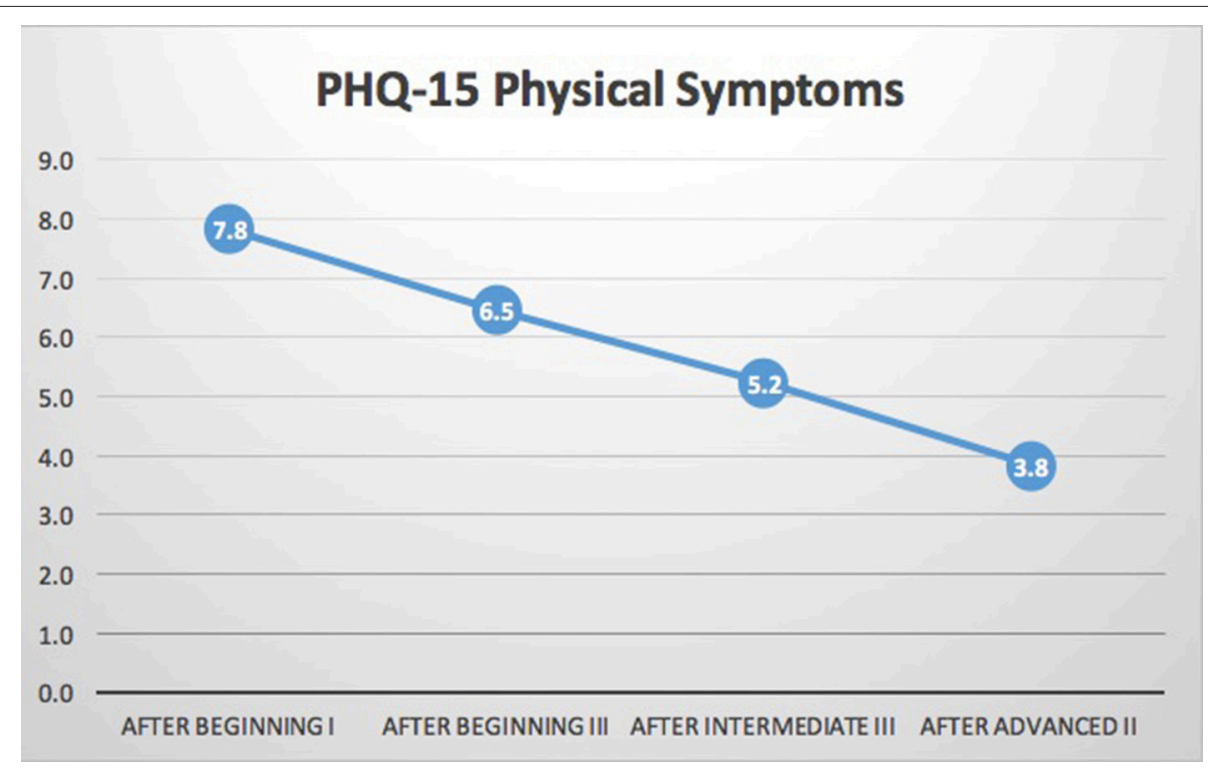

FIGURE 4 | PHQ-15 somatic symptoms. Results indicated significant reductions in somatic symptoms scale (PHQ-15) of the PHQ-SADS ( $p<0.001)$. While the data set is too small for clear trends to emerge, these data indicate a possible step-wise reduction in symptoms over the course of the training. The main focus of the training in each module is increased somatic awareness, capacity to regulate stress states when engaging in clinical work and increased skills in recognizing one's own indication of stress states through interoception and description of the state. The regular time for reflection and skills practice could be a driver for the change at each point of measurement.

chronic diseases (e.g., diabetes, hypertension, chronic pain, addiction, and stroke). However, trauma treatment also exposes individual professionals to the risk of vicarious traumatization, compassion fatigue and health related stress conditions. Some studies have indicated that compassion fatigue can lead to adverse changes in inflammatory cytokines and metabolic functioning and thereby increase risk for multiple negative health outcomes (Huffman, 2016). These health conditions also can impact treatment outcomes, as well as result in a significant burden on the healthcare system in the form of mental health worker attrition and of mental health workers who may become the "walking wounded." These treatment providers continue to show up to their jobs, but, due to the burden of stress, are compromised in their ability to actually get their jobs done.

A 2017 randomized study of Somatic Experiencing in individuals with PTSD trauma found significant reductions across multiple domains (Brom et al., 2017). One of the foundational concepts in the $\mathrm{SE}^{\circledR}$ model is that the increased clinical resilience is a likely outcome of training in the $\mathrm{SE}^{\circledR}$ model (Levine, 2010; Payne et al., 2015). Significant time and focus of the $\mathrm{SE}^{\circledR}$ training is directed toward increased clinician resilience. Further, the $\mathrm{SE}^{\circledR}$ model would indicate that increased clinician resilience would likely impact clinical outcomes (Levine, 2010).
This study is the first study to explore the impact of training in the $\mathrm{SE}^{\circledR}$ model on clinician resilience. Future research could explore issues of clinician case load and the question of impact of clinician resilience on clinical outcomes.

Another 2017 randomized control study of the $\mathrm{SE}^{\circledR}$ model was conducted with 91 pain patients found to have PTSD (Andersen et al., 2017). This study noted significant reduction of trauma symptoms and fear of movement as compared to controls. Patients with chronic pain have significantly higher rates of trauma than the general population. Physicians who treat pain patients report high levels of burnout and fatigue (Brennstuhl et al., 2015; Kroll and Macaulay, 2016). While the scope of this present study focuses on behavioral health professionals, increased clinician resilience in providers who treat pain patients could have a meaningful impact on current pain management teams (Kroll and Macaulay, 2016).

Results of the current study indicate that among individuals who completed the $\mathrm{SE}^{\circledR}$ training, there were significant reductions in psychological symptoms of anxiety, and also in somatic symptoms. Participants in the training showed an ongoing reduction in symptoms over the course of the three training years. This reduction was both statistically significant and clinically meaningful with both somatization symptoms 


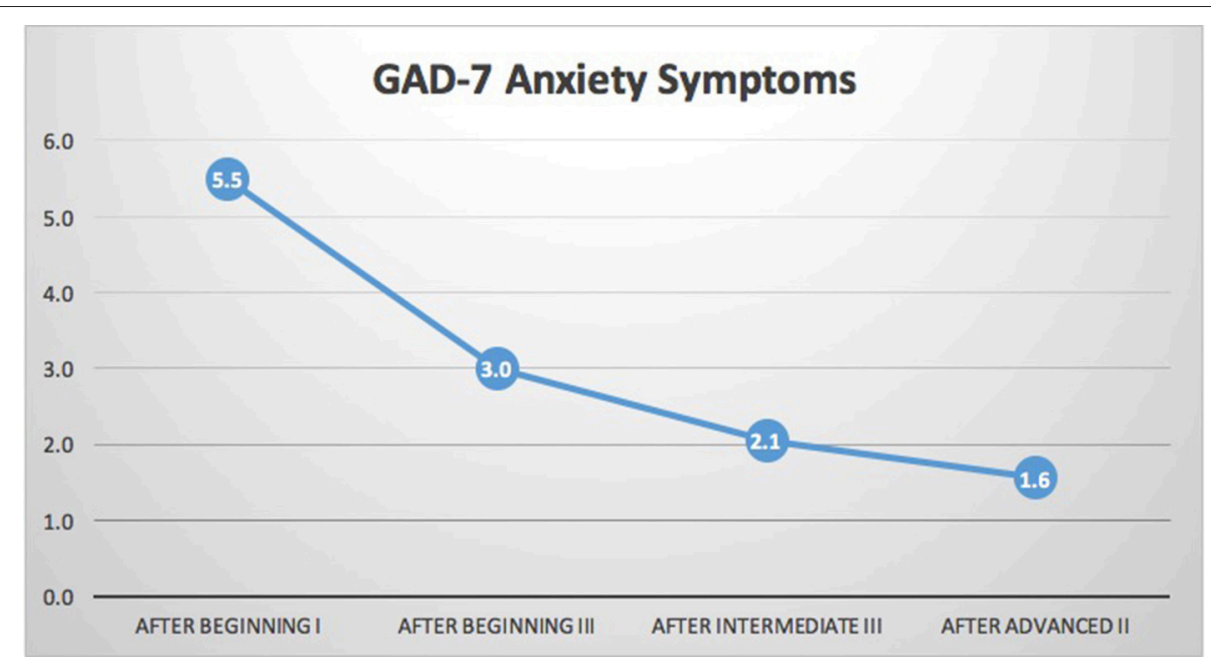

FIGURE 5 | GAD-7 generalized anxiety scale. Results indicated significant reductions in anxiety scale (GAD-7) of the PHQ-SADS ( $0<0.001)$. As stated above, the data set is too small for trends to be clearly identified but there appears to be a stable downward trend in symptoms of generalized anxiety. There is a slight flattening of the downward trend in the last two points of measure. It is likely that there are floor effects as the symptom level trends toward zero in the last two measures.

and symptoms of generalized anxiety dropping from mild to well below a clinical range. Notably, this was occurring at the same time that many of these individuals were treating individuals with symptoms of trauma and also working with $\mathrm{SE}^{\circledR}$ professionals on their own trauma histories. Results also indicate a significant increase in health-related and social quality of life among trainees. While both psychological quality of life and physical health related quality of life trended in a positive direction neither reached significance in this cohort. Further studies could elucidate the patterns driving these outcomes.

Psychological quality of life also appears to have increased at the final measurement compared to baseline. However, the change in psychological quality of life was not significant. Of course, improvement in psychological quality of life correlates with changes in psychological symptoms such as the ones found in this study. One possible moderating factor in this change is that the GAD-7 and PHQ-15 are measures of more specific domains likely to be impacted by the training in somatic self-regulation but the psychological quality of life measure used in this study was broader and less targeted to this outcome. Another possible driver could simply be that for this measure the study lacked the needed sample size for the effect size on this measure and a larger sample size could have more power and thus be more sensitive to the effects on psychological quality of life. Results of this pilot study support the importance of further study and assessment of real-world impacts, both on clinical outcomes and long-term clinician resilience, among professionals who treat trauma and trauma related conditions.

\section{LIMITATIONS}

The current study's design limits its generalizability. This is a small convenience sample of professionals and no control group. A possible enhancement would be to include a comparison group consisting of other professionals engaged in some other type of trauma training and a control group of professionals who have not had any form of trauma training. Another limitation impacting broader generalization of this study's findings was lack of data on the clinical setting of students in the study and their caseloads. An enhancement for future studies would be to include questions related to practice context and caseload to compare with changes in measures.

The current study had a problem with a high dropout rate. This is for several reasons: (1) no compensation of any sort was offered to the participants, (2) some students were forced to drop out of the training due to lack of funds. This is especially true for graduate students and interns. (3) some students, due to scheduling conflicts and lack of paid time off, are forced to wait an extra year to complete their $S E^{\circledR}$ training, (4) some students lose interest in studying trauma, and (5) the mathematical algorithm which needed to be calculated by participants in order to deidentify themselves proved to be daunting for some participants. Starting with a larger sample size and offering some incentive, as well as making the de-identification process easier would all help with this issue.

\section{DIRECTIONS FOR FUTURE RESEARCH}

Increased clinician resilience has profound implications for multiple professions and health/mental health providers. Future research would involve replicating these results with a larger sample size.

Other possibilities for future research would be using a control group of trauma therapists who do not undergo $S E^{\circledR}$ training, and examining clinician resilience vs. client outcomes. The impact of resiliency training on other high-risk profession groups such as substance abuse and suicide prevention counselors should be explored. Further research should collect data on the 
clinical setting of treating providers, caseloads and number of trauma patients.

Establishing a more direct causal pathway for these findings (reduction of anxiety, physical symptoms, and improvement in quality of life) with respect to resiliency, work satisfaction, and thus therapist retention within the trauma treatment field, would be a useful contribution to this area of study.

Resiliency is a complex heuristic that is still being clarified as to its scope. While further research is needed to confirm these findings, it is also possible, in future studies, to dissimilate the core components of increased resilience identified in this study. Researches exploring specific aspects of the training that mediate and moderate changes in resiliency could further support the field in helping to increase resilience, reduce the psychological impacts of trauma treatment, and improve outcomes in both therapists and clients. The measures used in this study are global measures of psychological health. There are multiple possible drivers for the changes identified in this study. While it was beyond the scope of this study to assess possible mechanisms of change the authors identified five candidates for future research. These are: (1) increased somatic mindfulness due to greater interoceptive awareness (Payne et al., 2015; Haase et al., 2016), (2) increased skills at resourcing or evoking a parasympathetic/calming response (Park et al., 2013), (3) increased acceptance and tolerance for intense sympathetically-mediated arousal states (Thompson et al., 2011; Nila et al., 2016), (4) changes in biological markers of sympathetic activation, ANS regulation and physiological health indicators (e.g., blood pressure, inflammatory cytokines or $24 \mathrm{~h}$ salivary cortisol), and (5) increased efficacy in clinicians, due to increased skills in addressing client symptoms (Shoji et al., 2016).

\section{REFERENCES}

Anda, R. F., Dong, M., Brown, D., Felitti, V., Gile, W., et al. (2009). Adverse childhood experiences and the risk of premature mortality. J. Prev. Med. 37, 389-396. doi: 10.1016/j.amepre.2009.06.021

Andersen, T. E., Lahav, Y., Ellegaard, H., and Manniche, C. (2017). A randomized controlled trial of brief Somatic Experiencing for chronic low back pain and comorbid post-traumatic stress disorder symptoms. Eur. J. Psychotraumatol. 8:1331108. doi: 10.1080/20008198.2017.1331108

Baird, S., and Jenkins, S. R. (2003). Vicarious traumatization, secondary traumatic stress, and burnout in sexual assault and domestic violence agency staff. Violence Vict. 18, 71-86. doi: 10.1891/vivi.2003. 18.1.71

Bell, H., Kulkarni, S., and Dalton, L. (2003). Organizational prevention of vicarious trauma. Famil. Soc. J. Contemp. Soc. Ser. 84, 463-470. doi: 10.1606/1044-3894.131

Brennstuhl, M. J., Tarquinio, C., and Montel, S. (2015). Chronic pain and PTSD: evolving views on their comorbidity. Perspect. Psychiatr. Care 51, 295-304. doi: $10.1111 /$ ppc. 12093

Brom, D., Stokar, Y., Lawi, C., Nuriel-Porat, V., Ziv, Y., Lerner, K., et al. (2017). Somatic experiencing for posttraumatic stress disorder: a randomized controlled outcome study. J. Trauma. Stress 30, 304-312. doi: 10.1002/jts.22189

Changaris, M. C. (2010). Assessing the Efficacy of Somatic Experiencing for Reducing Symptoms of Anxiety and Depresssion, Doctoral dissertation, John F. Kennedy University.

\section{ETHICS STATEMENT}

This study involved human subjects and was supervised by the private IRB, Independent, and Ethical Review Services under case number: 14033-03. All consent and data collection procedures were conducted in accordance with Declaration of Helsinki and guidelines outlined by IRB committee.

\section{AUTHOR CONTRIBUTIONS}

NW: Principal Investigator; NW, MC, and PS: Conceived and designed the study; NW: Fundraising; NW: IRB Interface; NW: Performed the study; NW, PS, and MC: Analyzed the data; NW, MC, and PS: Wrote the paper; NW, MC, and PS: Response to reviewers.

\section{FUNDING}

This study was primarily funded by the Principal Investigator, with additional crowd funding in 20-\$100 contributions by clinical practitioners in the Somatic Experiencing ${ }^{\circledR}$ community.

\section{ACKNOWLEDGMENTS}

We wish to acknowledge Dr. Mardi Crane, and Lela Carney, Lac, for their education and coaching during the early stages of research design, and the many private practitioners of Somatic Experiencing ${ }^{\circledR}$ for their financial contributions. Thank you also to the Foundation for Human Enrichment and David Berger, SEP, LMFT, RPT, for access to their Somatic Experiencing ${ }^{\circledR}$ training students for recruitment into this research.

Cieslak, R., Anderson, V., Bock, J., Moore, B. A., Peterson, A. L., and Benight, C. C. (2013). Secondary traumatic stress among mental health providers working with the military: prevalence and its work-and exposure-related correlates. J. Nerv. Ment. Dis. 201, 917-925. doi: 10.1097/NMD.000000000 0000034

Cocker, F., and Joss, N. (2016). Compassion fatigue among healthcare, emergency and community service workers: a systematic review. Int. J. Environ. Res. Public Health 13:618. doi: 10.3390/ijerph13060618

Craig, C. D., and Sprang, G. (2010). Compassion satisfaction, compassion fatigue, and burnout in a national sample of trauma treatment therapists. Anxiety Stress Coping 23, 319-339. doi: 10.1080/106158009030 85818

Engard, N. C. (2009). LimeSurvey. Available online at: http://limesurvey.org

Felitti, V. J., Anda, R. F., Nordenberg, D., Williamson, D. F., Spitz, A. M., Edwards, V., et al. (1998). Relationship of childhood abuse and household dysfunction to many of the leading causes of death in adults. The Adverse Childhood Experiences (ACE) study. Am. J. Prev. Med. 14, 245-258. doi: 10.1016/S0749-3797(98)00017-8

Fisher, J., and Ogden, P. (2009). "Sensorimotor psychotherapy," in Treating Complex Traumatic Stress Disorders, An Evidence Based Guide, eds C. A. Courtois and J. D. Ford (New York, NY: Guilford Press), 312-328.

Haase, L., Stewart, J. L., Youssef, B., May, A. C., Isakovic, S., Simmons, A. N., et al. (2016). When the brain does not adequately feel the body: links between low resilience and interoception. Biol. Psychol. 113, 37-45. doi: 10.1016/j.biopsycho.2015.11.004 
Han, C., Pae, C. U., Patkar, A. A., Masand, P. S., Kim, K. W., Joe, S. H., et al. (2009). Psychometric properties of the Patient Health Questionnaire-15 (PHQ-15) for measuring the somatic symptoms of psychiatric outpatients. Psychosomatics 50, 580-585. doi: 10.1176/appi.psy.50. 6.580

Heller, D. P., and Heller, L. (2004). Somatic Experiencing ${ }^{\circledR}$ in the Treatment of Automobile Accident Trauma. U.S. Assoc. Body Psycho Ther. J. 3, 42-52.

Horn, S. R., Charney, D. S., and Feder, A. (2016). Understanding resilience: new approaches for preventing and treating PTSD. Exp. Neurol. 284, 119-132. doi: 10.1016/j.expneurol.2016. 07.002

Huffman, M. C. (2016). Neuroendocrine and Psychological Factors Associated with Burnout, Compassion Fatigue, and Reduced Compassion Satisfaction in Mental Health Professionals. Doctoral dissertation, University of Nebraska at Omaha.

Johnson, W. B., Bertschinger, M., Snell, A. K., and Wilson, A. (2014). Secondary trauma and ethical obligations for military psychologists: preserving compassion and competence in the crucible of combat. Psychol. Serv. 11, 68-74. doi: $10.1037 / \mathrm{a} 0033913$

Kocalevent, R. D., Hinz, A., and Brähler, E. (2013). Standardization of a screening instrument (PHQ-15) for somatization syndromes in the general population. $B M C$ Psychiatry 13:91. doi: 10.1186/1471-244X-13-91

Kroenke, K., Spitzer, R., Williams, J., and Lowe, B. (2010a). The patient health questionnaire somatic, anxiety, and depressive symptom scales: a systematic review. Gen. Hosp. Psychiatry 32, 345-359. doi: 10.1016/j.genhosppsych.2010.03.006

Kroenke, K., Zhong, X., Theobald, D., Wu, J., Tu, W., and Carpenter, J. S. (2010b). Somatic symptoms in patients with cancer experiencing pain or depression: prevalence, disability, and health care use. Arch. Intern. Med. 170, 1686-1694. doi: 10.1001/archinternmed. 2010.337

Kroll, H., and Macaulay, T. (2016). A preliminary survey examining predictors of burnout in pain medicine physicians in the United States. Pain Phys. 19, E689-E696.

Leitch, M. L. (2007). Somatic Experiencing ${ }^{\circledR}$ treatment with tsunami survivors in thailand: broadening the scope of early intervention. Traumatology 13, 11-20. doi: $10.1177 / 1534765607305439$

Leitch, M. L., Vanslyke, J., and Allen, M. (2009). Somatic Experiencing ${ }^{\circledR}$ treatment with social service workers following hurricanes katrina and rita. Soc. Work 54, 9-18. doi: $10.1093 / \mathrm{sw} / 54.1 .9$

Levine, P. A. (2010). In an Unspoken Voice: How the Body Releases Trauma And Restores Goodness. Berkeley, CA: North Atlantic Books.

Li, M. Y., Yang, Y. L., Liu, L., and Wang, L. (2016). Effects of social support, hope and resilience on quality of life among Chinese bladder cancer patients: a cross-sectional study. Health Qual. Life Outcomes 14:73. doi: 10.1186/s12955-016-0481-z

Ménard, C., Pfau, M. L., Hodes, G. E., and Russo, S. J. (2016). Immune and neuroendocrine mechanisms of stress vulnerability and resilience. Neuropsychopharmacology $42, \quad 62-80$. doi: 10.1038/npp. 2016.90

Nila, K., Holt, D. V., Ditzen, B., and Aguilar-Raab, C. (2016). Mindfulnessbased stress reduction (MBSR) enhances distress tolerance and resilience through changes in mindfulness. Ment. Health Prevent. 4, 36-41. doi: 10.1016/j.mhp.2016.01.001

Park, E. R., Traeger, L., Vranceanu, A. M., Scult, M., Lerner, J. A., Benson, H., et al. (2013). The development of a patient-centered program based on the relaxation response: the Relaxation Response Resiliency Program (3RP). Psychosomatics 54, 165-174. doi: 10.1016/j.psym.2012.09.001
Parker, C., Doctor, R. M., and Selvam, R. (2008). Somatic therapy treatment effects with tsunami survivors. Traumatology 14, 103-109. doi: $10.1177 / 1534765608319080$

Payne, P., Levine, P. A., and Crane-Godreau, M. A. (2015). Somatic experiencing: using interoception and proprioception as core elements of trauma therapy. Front. Psychol. 6:93. doi: 10.3389/fpsyg.2015.00093

Pearlman, L., and Saakvitne, K. (1995). Trauma and the therapist: countertransference and vicarious traumatization in psychotherapy with incest survivors. Am. J. Clin. Hyp. 4:451. doi: 10.1080/00029157.1996.10403354

Pereira, J. A., Barkham, M., Kellett, S., and Saxon, D. (2016). The role of practitioner resilience and mindfulness in effective practice: a practice-based feasibility study. Adm. Policy Ment. Health Ment. Health Serv. Res. 44, 691-704. doi: 10.1007/s10488-016-0747-0

Power, M. (1998). Development of the World Health Organization WHOQOL-BREF quality of life assessment. Psychol. Med. 28, 551-558. doi: $10.1017 /$ S0033291798006667

Richardson, G. E., and Waite, P. J. (2002). Mental health promotion through resilience and resiliency education. Int. J. Emerg. Ment. Health 4, 65-75.

Sabin-Farrell, R., and Turpin, G. (2003). Vicarious traumatization: implications for the mental health of health workers? Clin. Psychol. Rev. 23, 449-480. doi: $10.1016 / \mathrm{S} 0272-7358(03) 00030-8$

Shoji, K., Cieslak, R., Smoktunowicz, E., Rogala, A., Benight, C. C., and Luszczynska, A. (2016). Associations between job burnout and self-efficacy: a meta-analysis. Anxiety Stress Coping 29, 367-386. doi: 10.1080/10615806.2015.1058369

Sprang, G., Clark, J. J., and Whitt-Woosley, A. (2007). Compassion fatigue, compassion satisfaction, and burnout: factors impacting a professional's quality of life. J. Loss Trauma 12, 259-280. doi: 10.1080/15325020701238093

The WHOQOL Group (1995). The World Health Organization quality of life assessment (WHOQOL): position paper from the world health organization. Soc. Sci. Med. 41, 1403-1409. doi: 10.1016/0277-9536(95)00112-K

Thompson, R. W., Arnkoff, D. B., and Glass, C. R. (2011). Conceptualizing mindfulness and acceptance as components of psychological resilience to trauma. Trauma Viol. Abuse 12, 220-235. doi: 10.1177/1524838011416375

Trippany, R. L., Kress, V. E. W., and Wilcoxon, S. A. (2004). Preventing vicarious trauma: what counselors should know when working with trauma survivors. J. Counsel. Dev. 82, 31-37. doi: 10.1002/j.1556-6678.2004.tb0 0283.x

Tugade, M. M., and Fredrickson, B. L. (2004). Resilient individuals use positive emotions to bounce back from negative emotional experiences. J. Pers. Soc. Psychol. 86:320. doi: 10.1037/0022-3514.86.2.320

van der Kolk, B. A. (1984). Post-Traumatic Stress Disorder: Psychological and Biological Sequelae. Washington, DC: American Psychiatric Pub Inc.

Whitehouse, B., and Heller, D. (2008). Heart Rate in trauma: patterns found in somatic experiencing and trauma resolution. Biofeedback 1, 24-29.

Conflict of Interest Statement: The authors declare that the research was conducted in the absence of any commercial or financial relationships that could be construed as a potential conflict of interest.

Copyright (๑) 2018 Winblad, Changaris and Stein. This is an open-access article distributed under the terms of the Creative Commons Attribution License (CC $B Y)$. The use, distribution or reproduction in other forums is permitted, provided the original author(s) and the copyright owner are credited and that the original publication in this journal is cited, in accordance with accepted academic practice. No use, distribution or reproduction is permitted which does not comply with these terms. 